celebration. His team is still analysing the data from the trial, and has not revealed how many bats were treated and released or how the controls fared. And even if this or the bacterial treatments are effective, they will be only short-term solutions. The fungus lingers on cave walls during the summer, and bats do not seem to develop immunity to it - so researchers would need to treat the animals every year to keep them from getting sick.

\section{STOPGAP SOLUTIONS}

Long-lasting solutions remain elusive. Some scientists hope to develop a vaccine, but have yet to work out how to trigger the animals' immune systems to destroy the pathogen, says Ken Field, an ecoimmunologist at Bucknell University in Lewisburg, Pennsylvania. Bats naturally produce antibodies to the fungus, but there is no evidence that these can help them to survive.

Other researchers are promoting a more radical long-term solution: altering airflow in mines where bats hibernate to make the sites less hospitable to the white-nose fungus. Places where bats survive infection tend to be relatively cool and dry. By opening new routes to the outside, researchers could cool and dehumidify the air in mines that are too warm and wet.

There is a chance that manipulating airflow could drive bats to abandon the habitat, says Kate Langwig, an ecologist at the UCSC. But she argues that it is worth a try, because at some sites the white-nose fungus kills about $90 \%$ of the bats present. In the first 5 years that it was present in the United States, the pathogen claimed more than 5.5 million animals.

In the meantime, the United States and Canada are developing and implementing strategies to coordinate work by scientists and by local and national governments - ranging from laboratory and field studies to efforts to prevent people from inadvertently spreading the fungus to pristine caves.

The plight of the bats is "stark - it's demoralizing", says Winifred Frick, an ecologist at the UCSC. "But I have hope in terms of the amount of creative energy and sense of dedication that people are putting forth on this problem. If there are solutions, we will find them."

\section{First glimpse of primordial stars}

\section{Astronomers claim to spot generation that seeded Universe.}

\section{BY ELIZABETH GIBNEY}

$\mathrm{S}$ ome of the first generation of stars, whose explosions breathed carbon, oxygen and other elements into the Universe, may have been glimpsed for the first time. The possibility comes as a pleasant surprise to astronomers, who did not expect to be able to spot these primordial objects with existing telescopes.

Primordial stars are theorized to be hundreds of times larger than the Sun, and made up only of pristine hydrogen, helium and traces of lithium left over from the Big Bang. The earliest specimens formed during the first few hundred million years of the Universe, living for only a few million years before exploding in supernovae that laid the seeds for the more element-rich stars to come. But they have never been seen.

A team led by David Sobral, an astronomer at the University of Lisbon, now reports that it may have spied a late-blooming cluster of such stars, in the brightest distant galaxy yet observed. The stars, seen as they were when the Universe was around 800 million years old (a mere $6 \%$ of its current age), seem to be primordial in composition - but, strangely, they also seem to reside in the same galaxy as some second-generation stars.

"Until now, work on these stars has been completely theoretical," says Sobral. "For the first time, we're starting to get observations that can test the many theories about these stars and begin to understand how they formed." His team's report has been posted on the preprint server arXiv and accepted for publication in The Astrophysical Journal (D. Sobral et al. Preprint at http://arxiv.org/ abs/1504.01734; 2015).

Seeing these ancient stars involves observing very distant galaxies. Their light takes billions

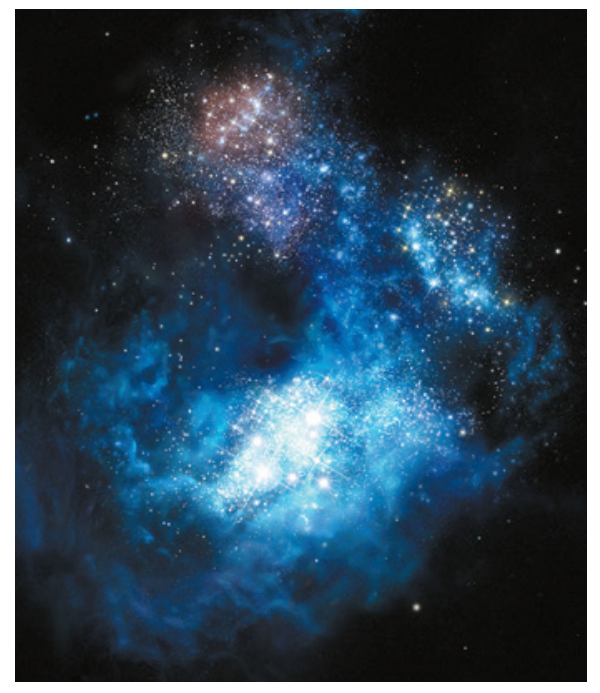

Artist's impression of galaxy CR7.

of years to reach us, and so shows the Universe in its earliest days. But the light is faint, making it difficult to spot. The short lifetimes of the first stars also makes them hard to find.

The surprise discovery emerged after Sobral and an international team of astronomers made a wide sweep of the sky using the Subaru Telescope on Mauna Kea in Hawaii. They used three further telescopes to peer into particularly bright galaxies, and found an intriguing signal from one that they named COSMOS Redshift 7. (The name was chosen so it could be abbreviated as CR7, to echo the nickname of Portuguese footballer Cristiano Ronaldo.)

The spectrum of light from CR7 showed evidence of ionized helium, which suggested that the source of the light was extremely hot. At such temperatures, any carbon and oxygen present should also have ionized, says Sobral. But there was no sign of these elements in

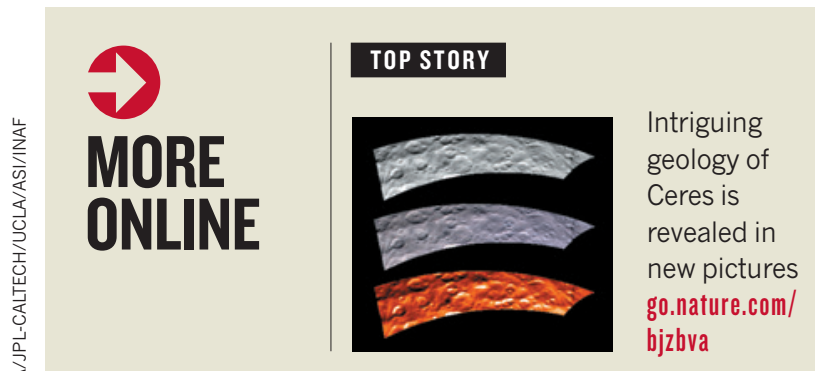

\section{MORE NEWS}

- Nuclear monitoring agency reaches out to scientists go.nature.com/bvtlme - Europe's first humans: what scientists do and don't know go.nature.com/usdonp

- Pope or International Energy Agency — who said what? go.nature.com/as3pr6

\section{NATURE PODCAST}

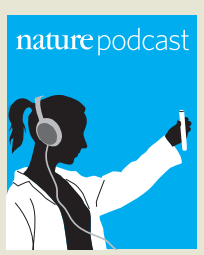

Antarctica's surprising biodiversity - and a new way to diagnose cancer early. nature.com/ nature/podcast 